\title{
Cavity-Mode Properties of Semiconductor Lasers Operating in Strong-Feedback Regime
}

\author{
Qin Zou ${ }^{1 *}$ and Abderrahmane Brézini ${ }^{2}$ \\ 1. Departement Electronique et Physique, Institut Mines-Telecom, UMR 5157 CNRS, 9 rue Charles Fourier, 91011 Evry Cedex, \\ France; CEA Saclay Nano-Innov, PC 176, Bât. 861, 91191 Gif sur Yvette Cedex, France \\ 2.Département de Chimie, Faculté des Sciences, Université d'Oran, BP 1524 Oran El M'Naouer, 31000 Oran, Algérie
}

\begin{abstract}
This paper investigates the modal properties of semiconductor lasers operating in the strong-feedback regime. Analytical expressions are developed based on an iterative travelling-wave model, which enable a complete and quantitative description of a compound cavity mode in its steady state. Additional information is provided about the physical inside into a compound laser system, such as a bifurcation diagram of the compound cavity modes for full variation range (from 0 to 1 ) of the external reflection coefficient and a more general shape for the diagram of photon density versus mode phase - this latter will reduce to the classical "ellipse" in the weak-feedback regime. It is shown that in the strong-feedback regime, a feedback laser is characterized by a small mode number and a high density of photons. This behavior confirms previous experimental observations, showing that beyond the coherence-collapse regime, the compound laser system could be re-stabilized, and that as a result power-enhanced low-noise stable laser operation with quasi-uniform pulsation is possible with external-mirror reflectivity close to 1 . Moreover, it is also shown that for a compound system operating in the strong-feedback regime, an anti-reflection treatment of a laser can significantly reduce its current threshold, and that in the absence of this treatment excitation of a minimum-linewidth mode with higher output power would be possible inside such a system. Finally, it is shown that in the weak-feedback regime except for a phase shift the iterative travelling-wave model will reduce to the Lang-Kobayashi model in cases where the product of the feedback rate and the internal round-trip time is much less than unity (that would mean in situations of as-cleaved lasers).
\end{abstract}

Key words: Semiconductor lasers, external optical feedback, compound cavity modes, weak-feedback regime, strong-feedback regime, coherence collapse, iterative travelling-wave model, Lang-Kobayashi model.

\section{Introduction}

It has been widely recognized that semiconductor lasers subject to external optical feedback can exhibit a large variety of interesting properties. A laser with external optical feedback builds an ideal physical system for analyzing and exploring typical phenomena encountered in a nonlinear time-delayed system, such as bifurcations, instability (and re-stability) thresholds and routes to deterministic chaos.

From the engineering point of view, external optical feedback can be used for improving the performance of a laser, such as line width narrowing, threshold lowering and intensity-noise reducing. External

\footnotetext{
*Corresponding author: Qin Zou, Ph.D., associate professor, research fields: optics and photonics. E-mail: qin.zou@telecom-sudparis.eu.
}

optical feedback, on the other hand, is also responsible for undesirable effects (for example, mode hopping, line width broadening and frequency destabilization) whose effective control is therefore essential for many laser-based applications, such as coherent communications, video recording and sensing systems.

Since the last three decades, intensive studies have been made aimed at better understanding the mechanism of the nonlinear dynamics of a compound laser system. In parallel with numerous experimental investigations, theoretical approaches have also been developed [1, 2]. Most of these approaches have been elaborated on the basis of the rate equations proposed by LK (Lang and Kobayashi) [3]. In the case of the WFB (Weak-feedback) regime of operation (feedback 
power ratio less than $-30 \mathrm{~dB}$ ), these approaches have been found to describe adequately various phenomena so far observed experimentally, such as the onset of CC (Coherence collapse) [4], the LFFs (Low-frequency fluctuations) [5] and the period-doubling route to deterministic chaos [6]. The LK rate equations have also been used to explain the physical mechanisms of the steady-state [7] and transient [8] LFFs, as well as of the chaotic itinerancy for the case of relatively strong feedback [7].

Originally, the LK rate equations were proposed to model a single-mode laser with weak feedback and large delays. When the reflectivity of the external reflecting surface is comparable with or greater than the laser facet reflectivity, strong feedback should be taken into account. In this case, the use of the LK model would no longer be justified. Thus, in order to describe the behavior of a feedback laser with arbitrary feedback levels, an ITW (Iterative traveling-wave) model was developed [9, 10]. By using this model, dynamic and noise properties of a laser subject to strong optical feedback were numerically investigated [11]. The ITW model predicts in particular a significant decrease of the intensity noise in the SFB (Strong-feedback) regime.

The behavior of a laser in the SFB regime was also analyzed by use of an improved model [12]. It was shown that, as in good agreement with experimental observations, in this state the compound laser system operates in uniform pulsation.

A TW (Traveling-wave) approach [13, 14] was proposed to model a feedback laser [15], where the system is described by partial differential equations for the electrical fields which counter-propagate along the longitudinal axis of the laser and are coupled through the usual carrier rate equation. A comparison has been made between the LK and TW models, with the emphasis on the stability analysis of the cavity modes in their continuous-wave states.

It was shown that the LK rate equations can be solved analytically by use of asymptotic methods [16].
In this approach, a laser operating in the WFB regime is regarded as a weakly-perturbed nonlinear dynamic system and the threshold of instability corresponds to the first Hopf bifurcation of the LK rate equations. An attempt has been made at interpreting experimental findings obtained with InAs/InP quantum-dash Fabry-Perot lasers by means of this approach, such as the onset of CC and the transition from the LFFs to SFB regimes [17].

This paper investigates the modal properties of a semiconductor laser under strong optical feedback by use of the ITW model. It may be considered as an extension of the contributions reported in $[11,18]$ and [12]. We give a deeper physical insight into a compound laser system by providing a quantitative description of a compound cavity mode, and discuss the similarities and the differences between the ITW and LK models. In Section 2, steady-state solutions will be derived for feedback-induced compound cavity modes. In Section 3, the properties of these modes will be discussed in details and compared with previous work. In Section 4, a quantitative comparison will be made between the ITW and LK models. We will show that in the WFB regime the ITW model will be reduced to the LK model in the case of as-cleaved lasers. Finally, Section 5 will summarize our conclusions.

\section{Iterative Traveling-Wave Model}

In this section, we outline briefly the formalism that we developed and examine some properties of a compound cavity mode in its steady state.

\subsection{Iterative Equation}

Consider the configuration of Fig. 1, a continuously operating (single-longitudinal-mode) laser diode is in resonance with an external Fabry-Perot cavity. It assumed that $r_{1}, r_{2}$ and $r_{3}$ are all real and dispersionless, and that $r_{1}=r_{2}$. For this three-mirror system, the dominant resonator is defined by the mirrors with reflection coefficients $r_{1}$ and $r_{3}$, and multiple round 


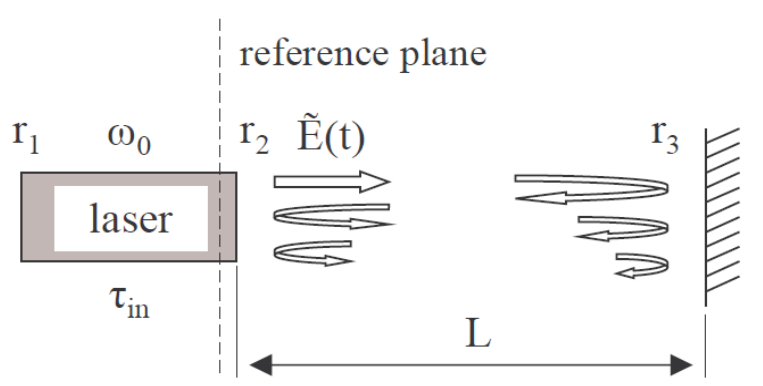

Fig. 1 Principle scheme of a single-mode laser diode with external optical feedback. $\omega_{0}$ : emission (angular) frequency of the solitary laser (in the absence of feedback); $\tau_{i n}$ : internal round-trip time; $r_{1}$ : reflection coefficient of the rear facet of the laser; $r_{2}$ : reflection coefficient of the front facet of the laser; $r_{3}$ : reflection coefficient of the external mirror; $\tilde{E}(t)$ :right-moving electric field passing through the laser front facet; $L$ : length of external cavity assumed empty.

trips of the light beam inside the external cavity should be in general taken into account for an arbitrary feedback level (i.e. $r_{3} \in[0,1]$ ).

For this compound laser system, the right-moving electric field $\tilde{E}(t)$, calculated at steps of the internal round-trip time $\tau_{\text {in }}$ (s) satisfies the following iterative equation $[11,18]$ :

$$
\begin{array}{r}
\widetilde{E}\left(t+\tau_{\text {in }}\right)=\frac{1}{r_{2}^{2}} \exp \left\{\frac{\tau_{i n} G_{N}}{2}(1+j \alpha)\left[N(t)-N_{t h}\right]\right\} \\
\times\left[\widetilde{E}(t)-\left(1-r_{2}^{2}\right) \sum_{k=0}^{M}\left(-r_{2} r_{3}\right)^{k} \times \widetilde{E}(t-k \tau) \exp \left(-j k \Delta_{0}\right)\right]
\end{array}
$$

In this equation, $G_{N}\left(\mathrm{~s}^{-1} \cdot \mathrm{m}^{3}\right)$ is the differential gain; $\alpha$ is the line width enhancement factor; $N(t)\left(\mathrm{m}^{-3}\right)$ is the carrier density and $N_{t h}$ (in $\mathrm{m}^{-3}$ ) is the threshold carrier density; $\tau$ (s) is the external round-trip time and $\Delta_{0}\left(\mathrm{rad}, \Delta_{0}=\omega_{0} \tau\right)$ is called the initial feedback phase which is associated with the emission frequency $\omega_{0}$ of the solitary laser operating just above threshold.

\subsection{Steady-State Solutions}

By inserting $\widetilde{E}(t)=\widetilde{E}_{0}(t) \exp (j \omega t)$ into Eq.(1) and considering steady-state solutions, we obtain the expression for the excess gain $\delta G$ (in $s^{-1}$ ):

$$
\delta G=\frac{-1}{\tau_{i n}} \ln \frac{(1-D)^{2}+E^{2}}{r_{2}^{4}}
$$

and that for the feedback phase $\Delta(\mathrm{rad}, \Delta=\omega \tau$ with $\omega$ : emission frequency of a possible mode):

$$
\operatorname{tg}\left(b_{2}-\Delta \frac{\tau_{\text {in }}}{\tau}\right)+\frac{E}{1-D}=0
$$

In the above two equations, $D$ (dimensionless), $E$ (dimensionless) and $b_{2}$ ( $\left.r a d\right)$, which are all functions of $\Delta_{0}$ and $\Delta$, can be written respectively as:

$$
\begin{gathered}
D=\left(1-r_{2}^{2}\right) \sum_{k=0}^{M}\left(-r_{2} r_{3}\right)^{k} \cos \left[k\left(\Delta_{0}+\Delta\right)\right] \\
E=\left(1-r_{2}^{2}\right) \sum_{k=0}^{M}\left(-r_{2} r_{3}\right)^{k} \sin \left[k\left(\Delta_{0}+\Delta\right)\right]
\end{gathered}
$$

and

$$
b_{2}=\frac{\tau_{\text {in }} \alpha}{2} \delta G=\frac{-\alpha}{2} \ln \frac{(1-D)^{2}+E^{2}}{r_{2}^{4}}
$$

Eqs. (2-6) are basic equations that we will use in the following for quantitatively describing the steady-state properties of a compound cavity mode (which may be called a CCM or simply a mode) generated under multiple-reflection configuration.

\subsection{Initial Feedback Phase}

Let us first examine the initial feedback phase $\Delta_{0}$. For a given compound structure, the phase $\Delta$ of a possible mode, being in dependence on $\Delta_{0}$, is determined through the so-called phase condition, namely Eq. (3).

It is known that under the consideration of low feedback levels $\left(r_{3}<<1\right)$, the phase equation developed from the LK rate equations has a simpler form:

$$
\Delta=\Delta_{0}-\gamma \tau[\alpha \cos (\Delta)+\sin (\Delta)]
$$

where, $\gamma\left(s^{-1}\right)$ is the feedback rate defined as usual $[1,2]$ :

$$
\gamma=\frac{\left(1-r_{2}^{2}\right) r_{3}}{r_{2} \tau_{\text {in }}}
$$

It can be seen from Eq. (7) that for the case of a solitary laser, an explicit expression for $\Delta_{0}$ cannot be obtained by setting $\gamma=0$ in this equation (giving rise to $\Delta_{0}=\Delta_{0}$ ). Two particular situations have been introduced and well studied where $\Delta$ is predefined 
and $\Delta_{0}$ is then determined from $\Delta$. The first situation corresponds to the "maximum-gain mode" defined by the condition $\Delta=0(\bmod 2 \pi)$. This gives rise to $\Delta_{0}=\gamma \tau \alpha(\bmod 2 \pi)$. In the second situation, the initial frequency remains unchanged $\left(\Delta=\Delta_{0}\right)$ and the related mode is called the "minimum-line width mode". We have thus $\Delta_{0}=-\operatorname{tg}^{-1}(\alpha)$.

In our case, the expression for $\Delta_{0}$ can be determined directly from the phase condition. So by putting $r_{3}=0$ and $M=0$ in Eqs. (4) and (5), we obtain $D=1-r_{2}^{2}, E=0$, and from Eq. (6), $b_{2}=0$. Eq. (3) becomes:

$$
\Delta_{0}=\frac{\tau}{\tau_{\text {in }}} m \pi=\beta m \pi \quad(m=0, \pm 1, \pm 2, \ldots)
$$

where, $\beta$ is the ratio between the external and internal round-trip times. This result implies that only discrete values of $\Delta_{0}$, which are authorized by the compound structure, and that these values are parameterized by the factor $\beta$, which is in general non integer and is much greater than 1 in most of the practical situations. We note that for both the "maximum-gain mode" and the "minimum-linewidth mode" exists a common solution in this paper, namely $\Delta_{0}=0$ (when $\alpha=0$ ), and that this corresponds to the zero-order solution ( $m=0)$ of Eq. (9). For simplicity, we will use in the following $\Delta_{0}=0$ as the value of the initial feedback phase.

\subsection{Modal Density of Photons}

From the standard rate equation for the carrier density $N[1,3]$ and by assuming a linear relation between the gain $G$ and $N$. the expression for the density of photons (or photon number) of a CCM was derived, which is denoted as $I\left(\mathrm{~m}^{-3}\right)$, Here $I$ is calculated directly from $\tilde{E}(t)$, It is written in steady state:

$$
I=\left|\widetilde{E}_{0}\right|^{2}=\frac{\left(J-\frac{N_{t h}}{\tau_{s}}\right)-\frac{1}{\tau_{s}} \cdot \frac{\delta G}{G_{N}}}{\tau_{p}^{-1}+\delta G}
$$

where, $\tau_{s}$ (in seconds) is the carrier life time, $\tau_{p}$ (in seconds) is the photon lifetime, and $J$ (in s ${ }^{-1} \cdot \mathrm{m}^{-3}$ ) is the injection current. With a large enough value of $J$, a finite number of modes can be excited. Each of these modes has its own current threshold denoted as $J_{t h}$, which, in certain situations, can be less than that of the solitary-laser mode, as will be shown in the following. By putting $I=0$ in Eq. (10), we obtain the expression of $J_{t h}$, which is written as:

$$
J_{t h}(\Delta)=\frac{1}{\tau_{s}}\left(\frac{\delta G(\Delta)}{G_{N}}+N_{t h}\right)
$$

For a solitary laser, $\delta G\left(\Delta_{0}\right)=0$, Eq. (11) reduces to the usual form $J_{t h}\left(\Delta_{0}\right)=N_{t h} / \tau_{s}$.

\section{Properties of Compound Cavity Modes}

\subsection{Bifurcation Diagram}

For a given compound structure, the phase condition determines the emission frequency of a possible mode. The phase $\Delta$ associated with this mode should satisfy Eq. (3), which is a transcendental equation. In general only numerical solutions are possible. The bifurcation diagrams of the CCMs for $r_{2}=0.2$ and $r_{2}=0.65$ are shown respectively in Fig. 2. The value of each point was obtained by a numerical solution of Eq. (3). Some parameter values used in the calculations are listed in Table 1.

Two particular regions in Fig. $2 \mathrm{~b}$ are presented in Fig. 3, where is found in (a) the shape predicted by the LK model as expected. We note that similar patterns can be obtained (not presented here) if the same regions in Fig. 2a are zoomed. It can be seen from Fig. 2 that when $r_{3}<r_{2}$, the number of modes increases progressively with $r_{3}$, as can be observed in a classical

Table 1 Some parameters and their values used in simulations.

\begin{tabular}{lll}
\hline Symbol & Value & Description \\
\hline$\tau_{\text {in }}$ & $9 \mathrm{ps}$ & Internal round-trip time \\
$\tau$ & $0.9 \mathrm{~ns}$ & External round-trip time \\
$\tau_{s}$ & $2 \mathrm{~ns}$ & Carrier lifetime \\
$G_{N}$ & $2 \times 10^{-12} \mathrm{~m}^{3} \cdot \mathrm{s}^{-1}$ & Differential gain \\
$N_{t h}$ & $10^{24} \mathrm{~m}^{-3}$ & Carrier density at threshold \\
$\Delta_{0}$ & $0 \mathrm{rad}$ & Initial feedback phase \\
$M$ & 100 & External round-trip number \\
\hline
\end{tabular}




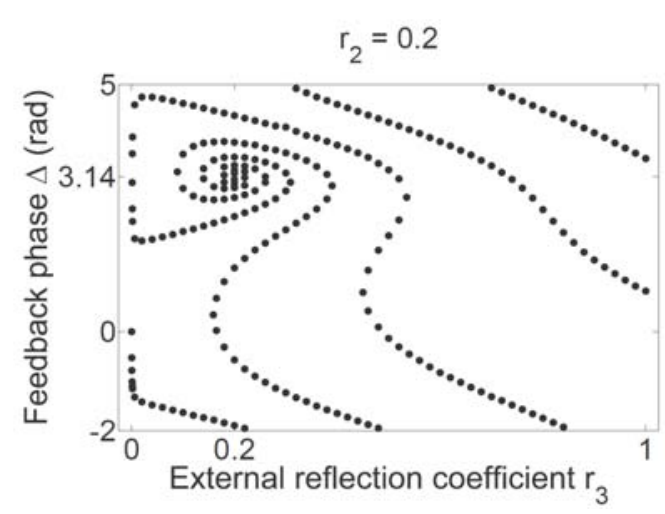

(a)

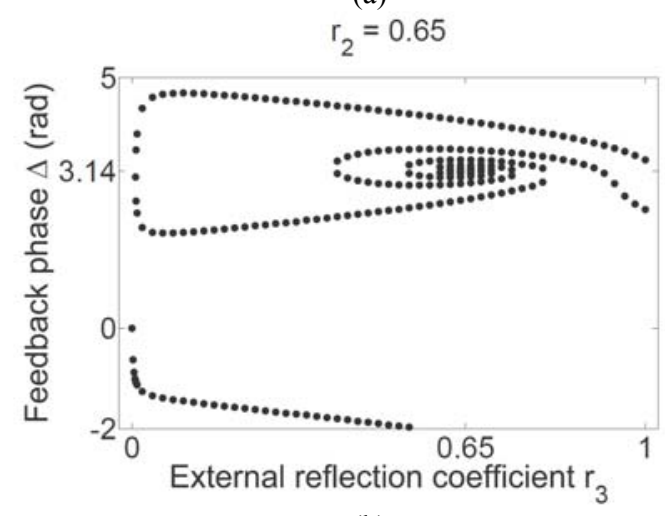

(b)

Fig. 2 Bifurcation diagram of the CCMs $(\alpha=5)$. (a) $r_{2}=0.2 ;$ (b) $r_{2}=0.65$. The values of the other parameters are listed in Table 1.

bifurcation pattern. The whirl-shape region around the point $\left(r_{2}, \pi\right)$ corresponds to the noisy $\mathrm{CC}$ region where many modes emerge. The maximum number of modes is attained at this critical point (Note that the excess gain $\delta G$ peaks at this point.). This situation corresponds to a symmetrical external cavity. For the case of $r_{3}>r_{2}$, the modes will disappear also progressively. Finally, the number of modes will become minimum in the SFB region when $r_{3}$ approaches unity. In this state, the compound system operates with significantly decreased RIN (Relative intensity noise), as observed experimentally in previous work $[19,20]$, indicating that beyond the noisy CC regime, the system could be re-stabilized, and that as a result pulsing operation of lasers with low intensity-noise level could be expected with external-mirror reflectivity comparable with 1 . Note that in a similar classification [12], the CC regime was called the chaotic regime.

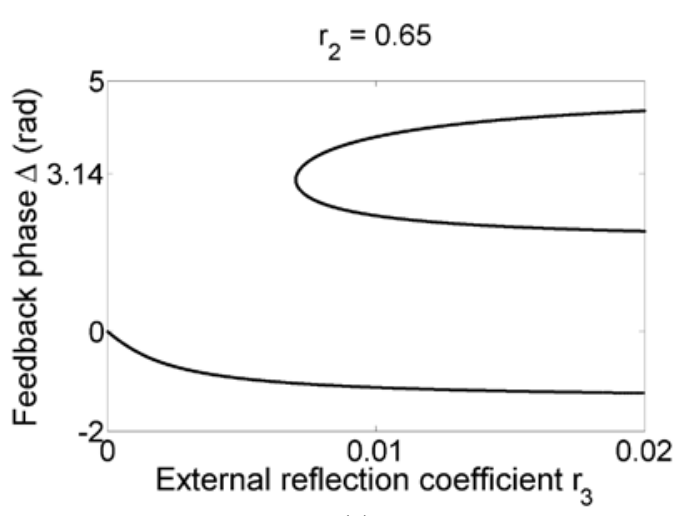

(a)

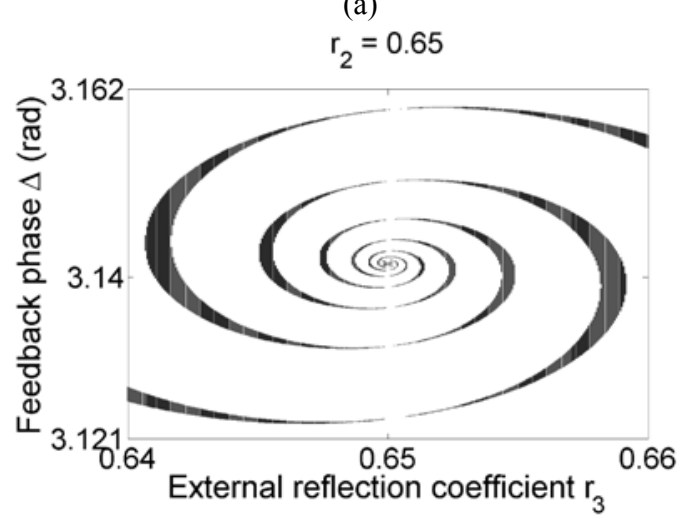

(b)

Fig. 3 Zoom on two particular regions in Fig. 2b. (a) Region which includes the WFB regime and the MFB (moderate-feedback) regime where the use of the LK model may still be justified; (b) Whirl-shape region near the critical point $\left(r_{2}, \pi\right)$, at which the maximum number of CCMs is found.

The transition from the CC to SFB regime has been observed with $1.57 \mu \mathrm{m} \quad \mathrm{InAs} / \mathrm{InP}$ quantum-dash Fabry-Perot lasers, when these lasers (with as-cleaved facets) were assessed for their tolerance to external optical feedback by using a free-space setup with a $0.5 \mathrm{~m}$-long external cavity [21]. In these experiments, the regime of strong feedback was attained for a pumping current of about $30 \mathrm{~mA}$ at a rather high feedback level ( $-1.2 \mathrm{~dB}$ in terms of power ratio) with a $600 \mu \mathrm{m}$-long as-cleaved laser. As can be seen from the measured RF (Radio frequency) spectra reported in this reference, the transition to the SFB regime is characterized in the frequency domain by a significant increase of each RF peak power around the relaxation oscillation frequency $(\sim 4 \mathrm{GHz})$ of the solitary laser. Such an increase has an effect more significant on lower frequency peaks and results as a consequence in 
an amplitude-enhanced quasi-uniform pulsation with frequency spacing of $300 \mathrm{MHz}[=c /(2 L)$, with $c$ : light velocity in vacuum].

This behavior manifested by a laser at a high feedback level can be quite well understood through the bifurcation diagram for the feedback phase $\Delta$. From Fig. 2, when $r_{3}$ is close to 1 , the number of possible modes becomes minimum for a feedback laser which is initially single-mode. Being in phase, these modes will interfere constructively to yield a pulsation at output. We note that the descriptions of this behavior in terms of the RIN spectrum and of the bifurcation diagram for the normalized carrier density are found in [11], showing clearly that high feedback levels can prevent a feedback laser from noisy output.

\subsection{Diagram of Photon Density versus Feedback} Phase

It is known that for a laser operating in the WFB (even in the MFB) regime, a common way to represent the CCMs at a fixed feedback level is through an ellipse showing the photon density $I$ Eq. (10) versus the feedback phase $\Delta$ Eq. (7), and that only a finite number of CCM points are possible which are all located on the ellipse [22].

At arbitrary feedback levels, the explicit relation between $I$ and $\Delta$ cannot be obtained straightforwardly, due to the transcendental form of the phase equation, Eq. (3). Thus, to analyze the $I-\Delta$ characteristic of a possible mode, it is more convenient to establish a diagram relating $I$ with a $\Delta$-dependent function denoted as $F(\Delta)$. We have from Eqs. (2) and (3):

$$
\Delta=F(\Delta)
$$

where,

$$
F(\Delta)=\frac{\tau \alpha \delta G(\Delta)}{2}-\frac{\tau}{\tau_{\text {in }}} \operatorname{tg}^{-1}\left[\frac{E(\Delta)}{D(\Delta)-1}\right]
$$

In this way, the $I-\Delta$ characteristic of a possible mode is described through the $I-F$ diagram, which is built with continuous variation of $\Delta$ for $F(\Delta)$. All the possible mode points, numerically calculated from Eq. (3), will be located on this curve, as indicated by Eq. (12).

Fig. 4a shows an example of $F$ versus $\Delta$ according to Eq. (13), for a laser with a symmetrical external cavity subject to relatively strong feedback $\left(r_{3}=r_{2}=0.2\right)$. We also plot the first ten mode points in the vicinity of the initial feedback phase $\Delta_{0}$ $\left(\Delta_{0}=0\right)$. The corresponding $I-F$ characteristic is illustrated in Fig. $4 \mathrm{~b}$.

It can be seen from this figure that the lower-order modes will give higher power output, and that if the maximum of the $I-F$ curve is referred to as the maximum-gain mode, the first possible mode ("-1" -order mode), being located the closest to the maximum-gain mode, will have the best chances to be dynamically stable.

As can be seen from Fig. 2, with the configuration of symmetrical external cavity a maximum number of possible modes can be generated inside the compound laser structure. Any mode (positive or negative order) with its $\Delta$ value close to $\pi$ or $-\pi$ will have a great $F$ value Fig. 4 a, which corresponds to a quasi-null value of the photon density Fig. $4 \mathrm{~b}$.

In Fig. 5, an example of the evolution of $I$ versus $F$ shape is given for various values of $r_{3}$, where two typical phenomena inside the $\mathrm{CC}$ regime are clearly shown: a banana-like shape and a shift, to positive $F$ values, of the CCM fixed points due to the inclusion of multiple reflections in the ITW model. Such a shape will become the most pronounced in the case of symmetrical external cavity. The perfect overlap of the two ellipses at $r_{3}=0.003$ means that in the WFB regime of operation $\left(r_{3}<<1\right)$, except for a phase constant, the ITW and LK models predict the same $I-\Delta$ characteristic. Moreover, when $r_{3}$ approaches 1 (very strong feedback), all the possible modes will have their photon densities greater than that of the solitary laser. 

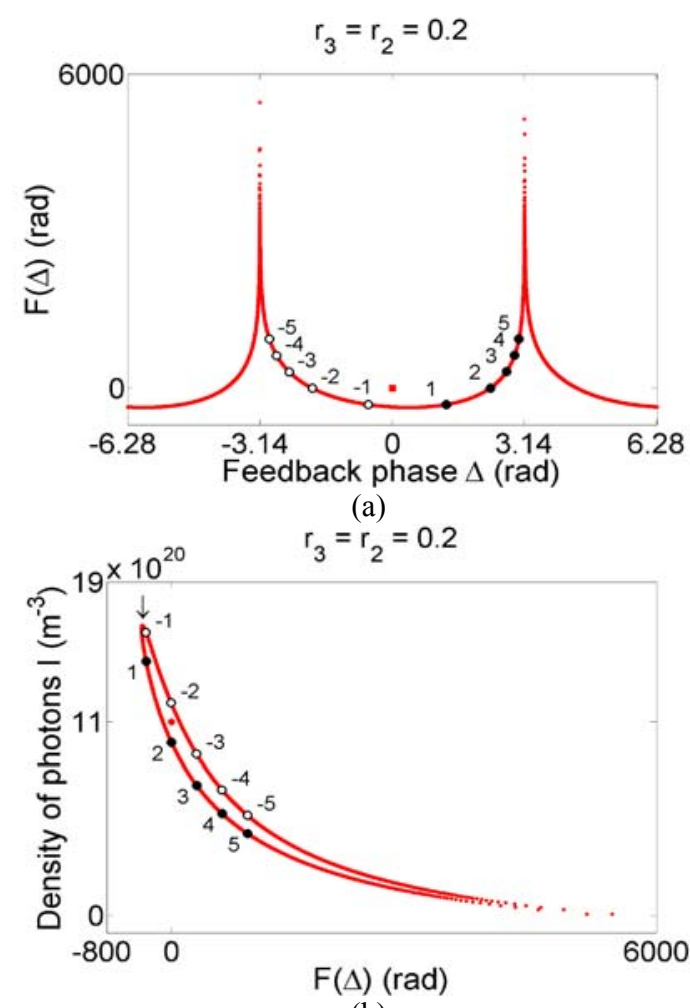

(b)

Fig. 4 (a) Variation of $F$ as a function of $\Delta$ (Eq. (13) )in the range of $-2 \pi \leq \Delta \leq 2 \pi$, for a symmetrical external cavity $\left(r_{3}=r_{2}=0.2\right)$ and the first ten mode points around $\Delta=0$ [numerical solutions of Eq. (3), $\alpha=5.5$ ]. The values of the other parameters are listed in Table 1. The red square refers to the solution of solitary laser; (b) Corresponding $I-F$ diagram $\left(\tau_{p}=2 p s\right)$. The maximum-gain mode is indicated by an arrow. The red dot refers to the solution of solitary laser.

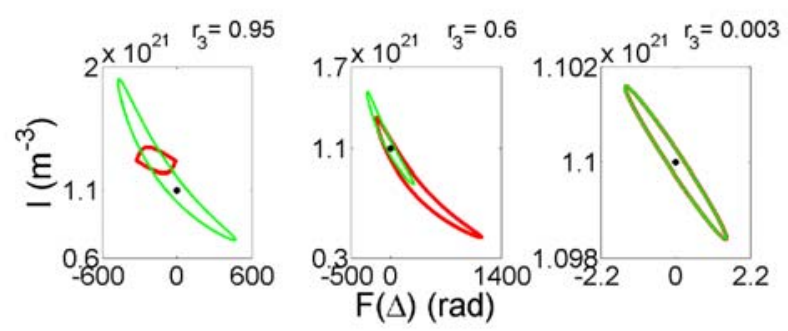

Fig. 5 Evolution of the CCM "ellipse" with decreasing of the external-mirror reflection coefficient $r_{3} \quad\left(r_{2}=0.65\right)$.

Red curves: from the ITW model; Green curves: from the LK model. The black dots refer to the solitary-laser solution.

\subsection{Reduction of Current Threshold and Enhancement of Output Power}

It is known that external optical feedback can have an effect on reduction of the current threshold, as observed with InAs/InP quantum-dash Fabry-Perot lasers [21] as well as with index-guided InGsAsP lasers [19].

For a given compound cavity mode $\Delta$, this effect can be characterized by:

$$
\delta J(\Delta)=J_{t h}\left(\Delta_{0}\right)-J_{t h}(\Delta)=\frac{-\delta G(\Delta)}{\tau_{s} G_{N}}
$$

So a mode $\Delta$ with its $\delta G<0$ will always have its current threshold reduced with respect to that of the solitary-laser mode $\Delta_{0}$. An example of the dependence of $\delta J$ on $r_{3}$ is shown in Fig. 6. We have considered the "- 1" -order mode for each combination of $r_{2}$ and $r_{3}$, and have chosen three typical values of $r_{2}: 0.5477$ for an as-cleaved facet $\left(r_{2}^{2}=0.3\right), \quad 0.2$ for a moderately-AR (anti-reflection)-coated facet, and 0.01 for a highly-AR-coated facet. The expression for the $r_{2}$-dependent photon lifetime $\tau_{p}$ is given by:

$$
\frac{1}{\tau_{p}}=\frac{c}{n_{g}}\left[\alpha_{i}-\frac{1}{l} \ln \left(r_{1} r_{2}\right)\right]
$$

where, $n_{g}$ is the group index of refraction, $\alpha_{i}$ (in $m^{-1}$ ) is the linear attenuation coefficient and $l$ (in $m$ ) is the length of internal cavity. For simplicity, we assume that $\alpha_{i}=0 \quad\left(r_{1}=r_{2}\right)$. so:

$$
\tau_{p}=\frac{-n_{g} l}{2 c \ln \left(r_{2}\right)}=\frac{-\tau_{\text {in }}}{4 \ln \left(r_{2}\right)}
$$

Fig. 6 shows that increasing the feedback level would not affect much the current threshold for an as-cleaved laser (a), and that maximum effect on threshold reduction is obtained when $r_{3}$ is close to unity for each of these three cases. The reduction effect becomes the most significant when the laser is highly AR-coated $\left(r_{2}=0.01\right)$ and operates in the SFB regime (c). Moreover, maximum effect on feedback-induced power enhancement can be expected with as-cleaved lasers (a).

We also find a phase shift, to negative values, of all these modes with respect to $\Delta_{0}$, assumed to be equal to zero. For the case of $r_{2}=0.5477$, we find that at $r_{3}=0.9$, the mode has its phase value close to zero: 


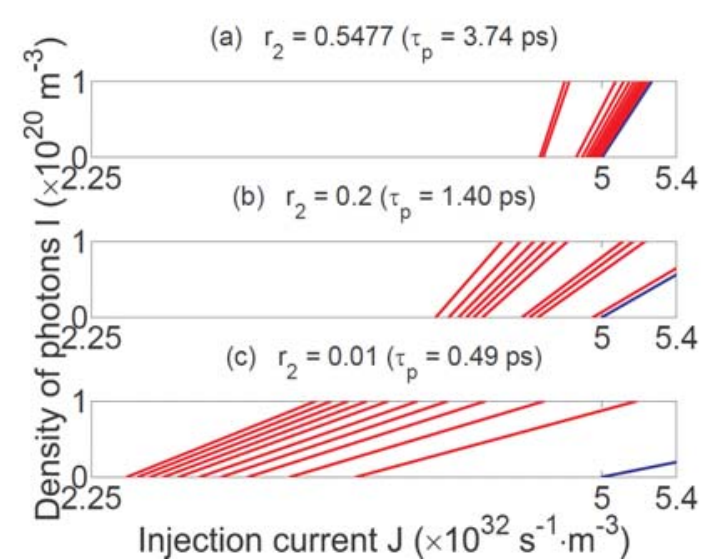

Fig. 6 Dependence, on the external reflection coefficient $r_{3}$, of the light versus current characteristic $(I-J)$, for three typical values of $r_{2}$. The solitary-laser $I-J$ curves $\left(J_{t h}\left(\Delta_{0}\right)=5 \times 10^{32} \mathrm{~s}^{-1} \cdot \mathrm{m}^{-3}\right.$ and $\left.r_{3}=0\right)$ are illustrated in blue. As $r_{3}$ increases from 0 to 1 (with increment of 0.1 ), each $I-J$ line will have its $\delta J$ as well as its slope $\left(\tau_{p}^{-1}+\delta G\right)^{-1}$ (Eq. (10)) increased monotonously.

$\Delta=-0.001184 \mathrm{rad}$. This result means that in the absence of AR treatment, a compound laser system operating in the SFB regime would be able to deliver a frequency same as that of its solitary laser $\left(\Delta=\Delta_{0}\right.$, minimum-line width mode) but with higher output power.

\section{Weak-Feedback Regime}

In this section, the condition will be derived under which, except for a phase term, the ITW model will reduce to the $\mathrm{LK}$ model in the WFB regime $\left(r_{3}<<1\right)$.

\subsection{General Case}

At low feedback levels, the light beam emitted by the solitary laser undergoes only a single round trip inside the external cavity $(M=1)$. Eqs. (4)-(6) are simplified respectively as:

$$
\begin{gathered}
D=\left(1-r_{2}^{2}\right)\left[1-r_{2} r_{3} \cos (\beta m \pi+\Delta)\right] \\
E=-r_{2} r_{3}\left(1-r_{2}^{2}\right) \sin (\beta m \pi+\Delta) \\
b_{2}=\frac{-\alpha}{2} \ln \left[1+\gamma^{2} \tau_{i n}^{2}+2 \gamma \tau_{\text {in }} \cos (\beta m \pi+\Delta)\right]
\end{gathered}
$$

where, Eq. (9) was used and the product $\gamma \tau_{\text {in }}$ (Eq. (8)) satisfies the following equation:

$$
\gamma \tau_{\text {in }}=\left(1-r_{2}^{2}\right) r_{3} / r_{2}
$$

By using Eq. (6), we obtain the expression for the steady-state excess gain:

$$
\delta G=-\tau_{\text {in }}^{-1} \ln \left[1+\gamma^{2} \tau_{\text {in }}^{2}+2 \gamma \tau_{\text {in }} \cos (\beta m \pi+\Delta)\right]
$$

and the phase condition:

$$
\operatorname{tg}\left(b_{2}-\frac{\Delta}{\beta}\right)=\frac{\gamma \tau_{\text {in }} \sin (\beta m \pi+\Delta)}{1+\gamma \tau_{\text {in }} \cos (\beta m \pi+\Delta)}
$$

It follows that both $\delta G$ and $\Delta$ of a possible mode depend on the product $\gamma \tau_{\text {in }}$. Two specific cases are discussed in the following.

\subsection{Case of as-Cleaved Lasers $\left(\gamma \tau_{\text {in }}<<1\right)$}

For a compound structure with an as-cleaved laser $\left(r_{2} \approx \sqrt{0.3}\right), \quad \gamma \tau_{i n}<<1$. For example, with $\tau_{\text {in }}=9 \mathrm{ps}$ and $r_{3}=0.005 \quad(-46 \mathrm{~dB}$ in terms of reflectivity), we have $\gamma \tau_{\text {in }}=6.4 \times 10^{-3}$. However, small values of $r_{2}$ due to AR coating on laser facets can make $\gamma \tau_{\text {in }}$ values comparable with 1 . Thus, with the same values of $\tau_{\text {in }}$ and $r_{3}$, we have with $r_{2}=0.01: \gamma \tau_{\text {in }}=0.5$.

In the case of $\gamma \tau_{\text {in }}<<1$, the excess gain and the phase condition have simpler forms. Eqs. $(19,21$ and 22) become hence (after the approximations $\ln (1+x) \approx x$ and $\operatorname{arctg}(x) \approx x)$ :

$$
\begin{gathered}
b_{2}=-\gamma \tau_{\text {in }} \alpha \cos (\beta m \pi+\Delta) \\
\delta G=-2 \gamma \cos (\beta m \pi+\Delta)
\end{gathered}
$$

$$
\Delta=\beta m \pi-\gamma \tau[\alpha \cos (\beta m \pi+\Delta)+\sin (\beta m \pi+\Delta)]
$$

Eqs. (24) and (25) clearly indicate that at low feedback levels except for a phase shift the ITW model will reduce to the LK model in the case of $\gamma \tau_{\text {in }}<<1$ (as-cleaved lasers). Note that the validity of the LK model is usually restricted by a less severe condition, namely $\gamma \tau_{\text {in }} \leq 1 / 2 \quad$ [22].

\subsection{Case of a Symmetrical External Cavity $\left(r_{3}=r_{2}\right)$}

For the three-mirror structure illustrated in Fig. 1, a 
lot of combinations are in principle possible for the values of $r_{2}$ and $r_{3}$, because of anti-reflection and high-reflection treatments on laser facets. Here, we are interested in a particular situation where $r_{3}=r_{2}$ (symmetrical external cavity).

Let us return to Fig. 2. As can be seen from this figure that, characterized by a whirl-like shape in the bifurcation pattern, the $\mathrm{CC}$ region is located around the point $\left(r_{2}, \pi\right)$. This phenomenon can be explained through Eqs. (22) and (20). Inside the CC region, many modes may exist. This corresponds to a great amplitude of the function

$\gamma \tau_{i n} \sin (\beta m \pi+\Delta) /\left[1+\gamma \tau_{i n} \cos (\beta m \pi+\Delta)\right]$ in Eq. (22). It can easily be shown that this function has its maximum $\quad A=\gamma \tau_{i n} / \sqrt{1-\gamma^{2} \tau_{i n}^{2}} \quad$ at $\Delta=\arccos \left(-\gamma \tau_{i n}\right)-\beta m \pi$. For a laser with $r_{2}^{2}<1$, $\gamma \tau_{\text {in }} \approx r_{3} / r_{2} \quad$ (Eq. (20)). This gives rise to $A \approx\left(r_{3} / r_{2}\right) / \sqrt{1-r_{3}^{2} / r_{2}^{2}}$. So $A$ is maximized when the external cavity becomes symmetrical $\left(r_{3}=r_{2}\right)$. This result implies that the whirl-shape region in the bifurcation pattern is in general centered at the critical point $\left(r_{2}, \pi-\beta m \pi\right)$, at which the maximum number of modes is obtained.

\subsection{Shift and Entrance of the Coherence-Collapse Region}

From the above discussions and Fig. 2, we see that decreasing $r_{2}$ values will make the $\mathrm{CC}$ region shifted to small values of $r_{3}$ and as a result will reduce the threshold for its entrance. This means that a laser with smaller $r_{2}$ values would be less resistant to external optical feedback, and that a compound system with a highly-AR-coated laser $\left(r_{2}<<1\right)$ could manifest instable chaotic behavior even at (very) low feedback levels. On the other hand, the same system could operate in pulsing regime at moderate feedback levels. These statements are found in good agreement with the results obtained in an early work [17], where it was shown that at the onset of $\mathrm{CC}$, the critical feedback level (in terms of the external-mirror reflection coefficient) $r_{3 \_C C}$ is related to $r_{2}$ by:

$$
r_{3_{-} C C}=\frac{-r_{2} \tau_{\text {in }} \Gamma_{R}}{\left(1-r_{2}^{2}\right) \sqrt{1+\alpha^{2}} \sin ^{2}\left(\frac{\omega_{R} \tau}{2}\right) \cos \left[\Delta_{0}-\operatorname{tg}^{-1}(\alpha)\right]}(26)
$$

In this equation, the term $\cos \left[\Delta_{0}-\operatorname{tg}^{-1}(\alpha)\right]$ should be negative and the relaxation oscillation frequency $\omega_{R}$ (in $\mathrm{s}^{-1}$ ) and the damping rate $\Gamma_{R}$ (in $\mathrm{s}^{-1}$ ) of the solitary laser are defined respectively as $\omega_{R}=\sqrt{G_{N} I / \tau_{p}}$ and $\Gamma_{R}=\left(\tau_{s}^{-1}+G_{N} I\right) / 2$.

\section{Conclusions}

This paper provides additional information about the physical insight into a feedback laser operating in the strong-feedback regime. Analytical expressions have been developed based on an iterative travelling-wave model, which enable a description in a rigorous way of a compound cavity mode in its steady state.

It is shown that with decreasing, from 1 , of the reflection coefficient of the external mirror, three regimes will emerge successively which can clearly be distinguished from the bifurcation diagram of the compound cavity modes: strong-feedback, coherence-collapse, and moderate-feedback regimes. This latter covers the weak-feedback regime where the use of the model of Lang and Kobayashi is entirely justified. It is found that, independently of feedback levels, the maximum number of modes is obtained when the external cavity becomes symmetrical. This state may cause a noisiest laser output. In the strong-feedback regime, a feedback laser is characterized by a small mode number and a high density of photons. This behavior confirms previous experimental observations, showing that beyond the coherence-collapse regime, the system could be re-stabilized, and that as a result power-enhanced low-noise laser operation with uniform pulsation is possible with external-mirror reflectivity close to 1 .

A more general way to represent the diagram of photon density versus feedback phase is proposed, whose shape will reduce to the classical "ellipse" in 
the weak-feedback regime.

For a compound system operating in the strong-feedback regime, an anti-reflection treatment of a laser can significantly reduce its current threshold, and that in the absence of this treatment, excitation of a minimum-linewidth mode with higher output power would be possible inside such a system in this paper.

A quantitative comparison is made between the iterative travelling-wave and Lang-Kobayashi models, showing that in the weak-feedback regime, these two models are identical (except for a phase constant) in cases where the product of the feedback rate and the internal round-trip time is much less than 1 - that would mean in situations of as-cleaved lasers.

Future investigations will include a detailed analysis of the coherence-collapse regime of anti-reflection-treated feedback lasers (which will more easily be coherence-collapsed and could be re-stabilized at moderate-feedback levels) by means of these two models.

\section{References}

[1] Tromborg, B., Osmundsen, J. H., Olesen, H. 1984. "Stability Analysis for a Semiconductor Laser in an External Cavity." IEEE Journal of Quantum Electronics QE-20 (9): 1023-32.

[2] Schunk, N. and Petermann, K. 1988. "Numerical Analysis of the Feedback Regimes for a Single-Mode Semiconductor Laser with External Feedback." IEEE Journal of Quantum Electronics 24 (7): 1242-7.

[3] Lang, R. and Kobayashi, K. 1980. "External Optical Feedback Effects on Semiconductor Injection Laser Properties.” IEEE Journal of Quantum Electronics QE-16 (3): 347-55.

[4] Lenstra, D., Verbeek, B. H. and Den Boef, A. J. 1985. "Coherence Collapse in Single-Mode Semiconductor Lasers due to Optical Feedback." IEEE Journal of Quantum Electronics 21 (6): 674-9.

[5] Fujiwara, M., Kubota, K. and Lang, R. 1981. "Low Frequency Intensity Fluctuation in Laser Diode with External Optical Feedback." Applied Physics Letters 38: 217-20.

[6] Ye, J., Li, H. and McInerney, J. G. 1993. "Period-Doubling Route to Chaos in a Semiconductor Laser with Weak Optical Feedback." Physical Review A 47 (3): 2249-52.

[7] Sano, T. 1994. "Antimode Dynamics and Chaotic
Itinerancy in the Coherence Collapse of Semiconductor Lasers with optical Feedback." Physical Review A 50 (3), 2719-26.

[8] Zamora-Munt, J., Masoller, C. and García-Ojalvo, J. 2010. "Transient Low-Frequency Fluctuations in Semiconductor Lasers with Optical Feedback." Physical Review A 81, 033820, 1-8.

[9] Sporleder, F. 1983. "Travelling Wave Line Model for Laser Diodes with External Optical Feedback." In Proceedings of the URSI International Symposium on Electromagnetic Theory, International Union of Radio Science, Brussels, 585-8.

[10] Mørk, J. 1989. Danish Center for Applied Mathematics and Mechanics. Rep. S48.

[11] Langley, L. N., Shore, K. A. and Mørk, J. 1994. "Dynamical and Noise Properties of Laser Diodes Subject to Strong optical Feedback." Optics Letters 19 (24): 2137-9.

[12] Abdulrhmann, S. G., Ahmed, M., Okamoto, T., Ishimori, W. and Yamada, M. 2003. "An Improved Analysis of Semiconductor Laser Dynamics under Strong Optical Feedback." IEEE Journal of Selected Topics in Quantum Electronics 9 (5), 1265-74.

[13] Carroll, J. E., Whiteaway, J. and Plumb, R. 1998. "Distributed Feedback Semiconductor Lasers." Institution of Electrical Engineers, London and SPIE Optical Engineering Press.

[14] Bandelow, U., Radziunas, M., Sieber, J. and Wolfrum, M. 2001. "Impact of Gain Dispersion on the Spatio-Temporal Dynamics of Multisection Lasers." IEEE Journal of Quantum Electronics 37 (2): 183-8.

[15] Radziunas, M., Wünsche, H. J., Krauskopf, B. and Wolfrum, M. 2006. "External Cavity Modes in Lang-Kobayashi and Traveling Wave Models." Proc. SPIE 6184, 6184X.

[16] Erneux, T. 2000 "Asymptotic Methods Applied to Semiconductor Laser Models.” Proc. SPIE 3944: 588-601.

[17] Zou, Q. and Azouigui, S. 2012. "Analysis of Coherence-Collapse Regime of Semiconductor Lasers under External Optical Feedback by Perturbation Method." Semiconductor Laser Diode Technology and Applications, Edition In Tech, Chapter 5: 71-86.

[18] Spencer, P. S., Mirasso, C. R. and Shore, K. A. 1998. "Effect of Strong Optical Feedback on Vertical-Cavity Surface-Emitting Lasers." IEEE Photonics Technology Letters 10 (2): 191-3.

[19] Temkin, H., Olsson, N. A., Abeles, J. H., Logan, R. A. and Panish, M. B. 1986. "Reflection Noise in Index-Guided InGaAsP Lasers." IEEE Journal of Quantum Electronics QE-22 (2): 286-93.

[20] Weiman, C. E. and Holberg, L. 1991. "Using Diode Lasers for Atomic Physics." Review of Scientific 
Instruments 62 (1)

[21] Azouigui, S., Kelleher, B., Hegarty, S. P., Huyet, G., Dagens, B. and Lelarge, F. et al.. 2007. "Coherence Collapse and Low-Frequency Fluctuations in Quantum-Dash Based Lasers Emitting at $1.57 \mu \mathrm{m} . "$
Optics Express 15 (21): 14155-62.

[22] Henry, C. H. and Kazarinov, R. F. 1986. "Instability of Semiconductor Lasers Due to Optical Feedback from Distant Reflectors." IEEE Journal of Quantum Electronics QE-22 (2): 294-301. 